\title{
Highly Enantioselective Dynamic Kinetic Resolution of 1,2-Diarylethanols by a Lipase-Ruthenium Couple
}

\author{
Mahn-Joo Kim*, Yoon Kyung Choi, Sol Kim, Daeho Kim, Kiwon Han, Soo-Byung \\ Ko and Jaiwook Park*
}

Department of Chemistry, Pohang University of Science and Technology (POSTECH), San-31 Hyojadong, Pohang 790-784, Korea

\section{Supporting Information}

\section{Representative procedure for kinetic resolution.}

To a solution of 1,2-diphenylethanol (2a, $60 \mathrm{mg}, 0.3 \mathrm{mmol})$, isopropenyl acetate $(99 \mu \mathrm{L}$, $0.9 \mathrm{mmol})$, and $\mathrm{K}_{2} \mathrm{CO}_{3}(60 \mathrm{mg}, 100 \mathrm{wt} \%)$ in anhydrous toluene $(1.0 \mathrm{~mL}, 0.3 \mathrm{M})$ was added PSL (vacuum-dried, $30 \mathrm{mg}, 50 \mathrm{wt} \%$ ) under an Ar atmosphere. The mixture was stirred at the ambient temperature for $12 \mathrm{~h}$. The base and enzyme were removed from reaction mixture by filtration through a celite. The solvents were evaporated under reduced pressure to give an oily mixture. For determining optical purities $\left(e_{p}\right.$ and $\left.e_{s}\right)$, it was dissolved in 2-propanol without further purification and then subject to the analysis by HPLC $\left(98 \% \mathrm{ee}_{\mathrm{p}}, 93 \% \mathrm{ee}_{\mathrm{s}}, 48 \%\right.$ conversion $)$.

\section{Determination of optical purities ( $\mathrm{ee}_{\mathrm{p}}$ and $\left.\mathrm{ee}_{\mathrm{s}}\right)$.}

The $\%$ ee $\mathrm{p}$ was determined with HPLC on a $(R, R)$ Whelk-O1 column [ $n$-hexane/2propanol $=95 / 5$; flow rate $=1 \mathrm{~mL} / \mathrm{min} ; \mathrm{UV}=217 \mathrm{~nm}$; retention times $=9.7 \mathrm{~min}(R), 6.1$ $\min (S)]$. The $\%$ ee $_{\mathrm{s}}$ was determined with HPLC on a Chiralcel OD-H column (Daicel) $[n$-hexane $/ 2$-propanol $=90 / 10$; flow rate $=1 \mathrm{~mL} / \mathrm{min} ; \mathrm{UV}=217 \mathrm{~nm}$; retention times $=$ $8.3 \min (R), 9.5 \min (S)]$.

\section{Representative procedure for dynamic kinetic resolution.}

To a $25-\mathrm{mL}$ flask equipped with a grease-free-high-vacuum stopcock were added 1,2diphenylethanol (2a, $39.7 \mathrm{mg}, 0.200 \mathrm{mmol}) 5$ (10.9 mg, $0.016 \mathrm{mmol}$ ), PSL (vacuumdried, $24.0 \mathrm{mg}, 120 \mathrm{mg} / \mathrm{mmol}$ substrate; specific activity, $2 \mathrm{U} / \mathrm{h}$ per $\mathrm{mg}$ of enzyme for 2a), and $\mathrm{K}_{2} \mathrm{CO}_{3}(27.6 \mathrm{mg}, 0.200 \mathrm{mmol})$ and the flask was filled with argon, followed by the addition of anhydrous toluene $(0.65 \mathrm{~mL})$ and isopropenyl acetate $(33 \mu \mathrm{L}, 0.30$ mmol). After being stirred at room temperature for 3 days, the reaction mixture was 
filtered on a celite, concentrated and purified by chromatography on a silica-gel column (ethyl acetate $/ n$-hexane $=1 / 9$ ) to give $(R)$-1-acetoxy-1,2-diphenylethane (3a, $47.0 \mathrm{mg}$, $0.196 \mathrm{mmol}$, 98\% yield, $98 \%$ ee).

(R)-1-Acetoxy-1,2-diphenylethane (3a): $[a]_{\mathrm{D}}{ }^{25}=+21.8\left(c=1.0, \mathrm{CHCl}_{3}, 98 \%\right.$ ee) (lit.: [ $a]_{\mathrm{D}}{ }^{15}=+14.0\left(c=0.5, \mathrm{CHCl}_{3}, 66 \%\right.$ ee. Ema, T.; Kageyama, M.; Korenaga, T.; Sakai, T. Tetrahedron: Asymmetry 2003, 14, 3943-3947.); ${ }^{1} \mathrm{H}$ NMR $\left(\mathrm{CDCl}_{3}, 300 \mathrm{MHz}\right) \delta 7.33$ $7.16(\mathrm{~m}, 8 \mathrm{H}), 7.10-7.08(\mathrm{~m}, 2 \mathrm{H}), 5.94(\mathrm{t}, J=6.5 \mathrm{~Hz}, 1 \mathrm{H}), 3.19\left(\mathrm{dd}, J_{1}=13.7 \mathrm{~Hz}, J_{2}=\right.$ $7.9 \mathrm{~Hz}, 1 \mathrm{H}), 3.04\left(\mathrm{dd}, J_{1}=13.7 \mathrm{~Hz}, J_{2}=6.0 \mathrm{~Hz}, 1 \mathrm{H}\right), 2.00(\mathrm{~s}, 3 \mathrm{H}) ;{ }^{13} \mathrm{C} \mathrm{NMR}\left(\mathrm{CDCl}_{3}\right.$, $75 \mathrm{MHz}) \delta 170.1,140.1,137.0,129.5,128.4,128.2$, 128.0, 126.7, 126.6, 76.6, 43.0, 21.2; HRMS (EI) $\mathrm{C}_{16} \mathrm{H}_{16} \mathrm{O}_{2}$ calcd $240.1150\left(\mathrm{M}^{+}\right)$, found 240.1153; The \% ee was determined by HPLC on a $(R, R)$ Whelk-O1 [ $n$-hexane/2-propanol $=95 / 5$; flow rate $=1$ $\mathrm{mL} / \mathrm{min} ; \mathrm{UV}=217 \mathrm{~nm}$; retention times $=9.7 \min (R), 6.1 \mathrm{~min}(S)]$.

(R)-1-Acetoxy-1-phenyl-2-(3-methylphenyl)-ethane (3b): $[a]_{\mathrm{D}}{ }^{25}=+20.2(c=1.0$, $\mathrm{CHCl}_{3}, 98 \%$ ee); ${ }^{1} \mathrm{H} \mathrm{NMR}\left(\mathrm{CDCl}_{3}, 300 \mathrm{MHz}\right) \delta 7.34-7.25(\mathrm{~m}, 5 \mathrm{H}), 7.12(\mathrm{t}, J=7.5 \mathrm{~Hz}$, $1 \mathrm{H}), 7.00(\mathrm{~d}, J=7.5 \mathrm{~Hz}, 1 \mathrm{H}), 6.93-6.87(\mathrm{~m}, 2 \mathrm{H}), 5.92(\mathrm{t}, J=6.4 \mathrm{~Hz}, 1 \mathrm{H}), 3.15\left(\mathrm{dd}, J_{1}=\right.$ $\left.13.7 \mathrm{~Hz}, J_{2}=8.0 \mathrm{~Hz}, 1 \mathrm{H}\right), 3.00\left(\mathrm{dd}, J_{1}=13.7 \mathrm{~Hz}, J_{2}=6.0 \mathrm{~Hz}, 1 \mathrm{H}\right), 2.29(\mathrm{~s}, 3 \mathrm{H}), 2.01(\mathrm{~s}$, $3 \mathrm{H}) ;{ }^{13} \mathrm{C} \mathrm{NMR}\left(\mathrm{CDCl}_{3}, 75 \mathrm{MHz}\right) \delta 170.1,140.2,137.8,137.0,130.3,128.3,128.1$, 127.9, 127.3, 126.6, 126.5, 76.7, 42.9, 21.3, 21.2; HRMS (EI) $\mathrm{C}_{17} \mathrm{H}_{18} \mathrm{O}_{2}$ calcd 254.1307 $\left(\mathrm{M}^{+}\right)$, found 254.1309; The \% ee was determined by HPLC on a $(R, R)$ Whelk-O1 $[n$ hexane $/ 2$-propanol $=95 / 5$; flow rate $=1 \mathrm{~mL} / \mathrm{min} ; \mathrm{UV}=217 \mathrm{~nm}$; retention times $=9.9$ $\min (R), 6.2 \min (S)]$.

(R)-1-Acetoxy-1-phenyl-2-(3-methoxyphenyl)-ethane $(\mathbf{3 c}):[a]_{\mathrm{D}}{ }^{25}=+16.4$ (c $=1.0$, $\mathrm{CHCl}_{3}, 97 \%$ ee); ${ }^{1} \mathrm{H} \mathrm{NMR}\left(\mathrm{CDCl}_{3}, 300 \mathrm{MHz}\right) \delta 7.34-7.25(\mathrm{~m}, 5 \mathrm{H}), 7.15(\mathrm{t}, J=7.9 \mathrm{~Hz}$, $1 \mathrm{H}), 6.75-6.68(\mathrm{~m}, 2 \mathrm{H}), 6.62(\mathrm{bs}, 1 \mathrm{H}), 5.94(\mathrm{t}, J=6.5 \mathrm{~Hz}, 1 \mathrm{H}), 3.73(\mathrm{~s}, 3 \mathrm{H}), 3.17\left(\mathrm{dd}, J_{1}\right.$ $\left.=13.7 \mathrm{~Hz}, J_{2}=7.8 \mathrm{~Hz}, 1 \mathrm{H}\right), 3.02\left(\mathrm{dd}, J_{1}=13.7 \mathrm{~Hz}, J_{2}=6.1 \mathrm{~Hz}, 1 \mathrm{H}\right), 2.02(\mathrm{~s}, 3 \mathrm{H}) ;{ }^{13} \mathrm{C}$ $\mathrm{NMR}\left(\mathrm{CDCl}_{3}, 75 \mathrm{MHz}\right) \delta 170.1,159.4,140.1,138.5,129.2,128.6,128.4,128.0,126.6$, 121.9, 115.0, 112.2, 76.5, 55.1, 43.0, 21.2; HRMS (EI) $\mathrm{C}_{17} \mathrm{H}_{18} \mathrm{O}_{3}$ calcd 270.1256 (M ${ }^{+}$), found 270.1258; The \% ee was determined by HPLC on a $(R, R)$ Whelk-O1 $[n$-hexane/2propanol $=90 / 10$; flow rate $=1 \mathrm{~mL} / \mathrm{min} ; \mathrm{UV}=217 \mathrm{~nm}$; retention times $=10.4 \mathrm{~min}(R)$, $6.9 \min (S)]$.

(R)-1-Acetoxy-1-phenyl-2-(3-phenoxy)-ethane (3d): $[a]_{\mathrm{D}}{ }^{25}=-7.5\left(c=1.0, \mathrm{CHCl}_{3}\right.$, $96 \%$ ee); ${ }^{1} \mathrm{H} \mathrm{NMR}\left(\mathrm{CDCl}_{3}, 300 \mathrm{MHz}\right) \delta 7.33-7.18(\mathrm{~m}, 8 \mathrm{H}), 7.08(\mathrm{t}, J=7.3 \mathrm{~Hz}, 1 \mathrm{H})$, 6.92-6.84 (m, 4H), $6.73(\mathrm{bs}, 1 \mathrm{H}), 5.91(\mathrm{t}, J=6.7 \mathrm{~Hz}, 1 \mathrm{H}), 3.17\left(\mathrm{dd}, J_{1}=13.7 \mathrm{~Hz}, J_{2}=\right.$ $7.9 \mathrm{~Hz}, 1 \mathrm{H}), 3.01\left(\mathrm{dd}, J_{1}=13.7 \mathrm{~Hz}, J_{2}=6.0 \mathrm{~Hz}, 1 \mathrm{H}\right), 2.00(\mathrm{~s}, 3 \mathrm{H}) ;{ }^{13} \mathrm{C} \mathrm{NMR}\left(\mathrm{CDCl}_{3}\right.$, $75 \mathrm{MHz}) \delta 170.0,157.3,157.0,139.8,139.0$, 129.7, 129.6, 128.4, 128.0, 126.6, 124.5, 123.1, 120.2, 118.7, 117.4, 76.5, 42.8, 21.2; HRMS (EI) $\mathrm{C}_{22} \mathrm{H}_{20} \mathrm{O}_{3}$ calcd $332.1412\left(\mathrm{M}^{+}\right)$, 
found 332.1410; The \% ee was determined by HPLC on a $(R, R)$ Whelk-O1 [ $n$-hexane/2propanol $=95 / 5$; flow rate $=1 \mathrm{~mL} / \mathrm{min} ; \mathrm{UV}=217 \mathrm{~nm}$; retention times $=13.0 \mathrm{~min}(R)$, $7.1 \min (S)]$.

(R)-1-Acetoxy-1-phenyl-2-(4-fluorophenyl)-ethane $(3 \mathrm{e}):[a]_{\mathrm{D}}{ }^{25}=+26.4 \quad(c=1.0$, $\mathrm{CHCl}_{3}, 97 \%$ ee); ${ }^{1} \mathrm{H} \mathrm{NMR}\left(\mathrm{CDCl}_{3}, 300 \mathrm{MHz}\right) \delta 7.31-7.23(\mathrm{~m}, 5 \mathrm{H}), 7.05-7.01(\mathrm{~m}, 2 \mathrm{H})$, 6.94-6.88 (m, 2H), $5.90(\mathrm{t}, J=6.4 \mathrm{~Hz}, 1 \mathrm{H}), 3.16\left(\mathrm{dd}, J_{1}=13.8 \mathrm{~Hz}, J_{2}=7.7 \mathrm{~Hz}, 1 \mathrm{H}\right)$, $3.02\left(\mathrm{dd}, J_{1}=13.8 \mathrm{~Hz}, J_{2}=6.2 \mathrm{~Hz}, 1 \mathrm{H}\right), 2.02(\mathrm{~s}, 3 \mathrm{H}) ;{ }^{13} \mathrm{C} \mathrm{NMR}\left(\mathrm{CDCl}_{3}, 75 \mathrm{MHz}\right) \delta$ $170.1,163.3,139.7,132.7,132.6,131.0,130.9$, 128.4, 128.1, 126.6, 115.2, 114.9, 76.5, 42.1, 21.1; HRMS (EI) $\mathrm{C}_{16} \mathrm{H}_{15} \mathrm{O}_{2} \mathrm{~F}$ calcd $258.1056\left(\mathrm{M}^{+}\right)$, found 258.1050; The \% ee was determined by HPLC on a $(R, R)$ Whelk-O1 $[n$-hexane/2-propanol $=95 / 5$; flow rate $=1$ $\mathrm{mL} / \mathrm{min} ; \mathrm{UV}=217 \mathrm{~nm}$; retention times $=9.3 \min (R), 5.8 \mathrm{~min}(S)]$.

(R)-1-Acetoxy-1-phenyl-2-p-tolyl-ethane (3f): $[a]_{\mathrm{D}}{ }^{25}=+18.8\left(c=1.0, \mathrm{CHCl}_{3}, 97 \%\right.$ ee); ${ }^{1} \mathrm{H}$ NMR $\left(\mathrm{CDCl}_{3}, 300 \mathrm{MHz}\right) \delta 7.33-7.24(\mathrm{~m}, 5 \mathrm{H}), 7.05-6.96(\mathrm{~m}, 4 \mathrm{H}), 5.91(\mathrm{t}, J=7.4 \mathrm{~Hz}$, $1 \mathrm{H}), 3.15\left(\mathrm{dd}, J_{1}=13.7 \mathrm{~Hz}, J_{2}=7.9 \mathrm{~Hz}, 1 \mathrm{H}\right), 3.00\left(\mathrm{dd}, J_{1}=13.7 \mathrm{~Hz}, J_{2}=6.0 \mathrm{~Hz}, 1 \mathrm{H}\right)$, $2.31(\mathrm{~s}, 3 \mathrm{H}), 2.00(\mathrm{~s}, 3 \mathrm{H}) ;{ }^{13} \mathrm{C} \mathrm{NMR}\left(\mathrm{CDCl}_{3}, 75 \mathrm{MHz}\right) \delta 170.1,140.2,136.0,133.9$, 129.4, 129.0, 128.9, 128.3, 127.9, 126.6, 76.7, 42.5, 21.2, 21.1; HRMS (EI) $\mathrm{C}_{17} \mathrm{H}_{18} \mathrm{O}_{2}$ calcd $254.1307\left(\mathrm{M}^{+}\right)$, found 254.1305 ; The \% ee was determined by HPLC on a $(R, R)$ Whelk-O1 $[n$-hexane/2-propanol $=95 / 5$; flow rate $=1 \mathrm{~mL} / \mathrm{min} ; \mathrm{UV}=217 \mathrm{~nm}$; retention times $=10.2 \min (R), 6.2 \min (S)]$.

(R)-1-Acetoxy-1-phenyl-2-(4-methoxyphenyl)-ethane $(\mathbf{3 g}):[a]_{\mathrm{D}}{ }^{25}=+15.3 \quad(c=1.0$, $\mathrm{CHCl}_{3}, 99 \%$ ee); ${ }^{1} \mathrm{H} \mathrm{NMR}\left(\mathrm{CDCl}_{3}, 300 \mathrm{MHz}\right) \delta 7.33-7.25(\mathrm{~m}, 5 \mathrm{H}), 7.00(\mathrm{~d}, J=8.5 \mathrm{~Hz}$, 2H), $6.77(\mathrm{~d}, J=8.5 \mathrm{~Hz}, 2 \mathrm{H}), 5.89(\mathrm{t}, J=7.0 \mathrm{~Hz}, 1 \mathrm{H}), 3.77(\mathrm{~s}, 3 \mathrm{H}), 3.13\left(\mathrm{dd}, J_{1}=13.8\right.$ $\left.\mathrm{Hz}, J_{2}=7.7 \mathrm{~Hz}, 1 \mathrm{H}\right), 2.99\left(\mathrm{dd}, J_{1}=13.8 \mathrm{~Hz}, J_{2}=6.2 \mathrm{~Hz}, 1 \mathrm{H}\right), 2.02(\mathrm{~s}, 3 \mathrm{H}) ;{ }^{13} \mathrm{C} \mathrm{NMR}$ $\left(\mathrm{CDCl}_{3}, 75 \mathrm{MHz}\right) \delta 170.1,158.3,140.1,130.5,129.0,128.3,127.9,126.6,113.6,76.8$, 55.2, 42.1, 21.2; HRMS (EI) $\mathrm{C}_{17} \mathrm{H}_{18} \mathrm{O}_{3}$ calcd 270.1256 $\left(\mathrm{M}^{+}\right)$, found 270.1261; The \% ee was determined by HPLC on a $(R, R)$ Whelk-O1 $[n$-hexane/2-propanol $=90 / 10$; flow rate $=1 \mathrm{~mL} / \mathrm{min} ; \mathrm{UV}=217 \mathrm{~nm}$; retention times $=9.8 \min (R), 6.7 \mathrm{~min}(S)]$.

(R)-1-Acetoxy-1-phenyl-2-(4-isopropylphenyl)-ethane $(\mathbf{3 h}):[a]_{\mathrm{D}}{ }^{25}=+21.9(c=1.0$, $\mathrm{CHCl}_{3}, 99 \%$ ee); ${ }^{1} \mathrm{H} \mathrm{NMR}\left(\mathrm{CDCl}_{3}, 300 \mathrm{MHz}\right) \delta 7.34-7.26(\mathrm{~m}, 5 \mathrm{H}), 7.10(\mathrm{~d}, J=8.0 \mathrm{~Hz}$, 2H), $7.03(\mathrm{~d}, J=8.0 \mathrm{~Hz}, 2 \mathrm{H}), 5.91(\mathrm{t}, J=6.1 \mathrm{~Hz}, 1 \mathrm{H}), 3.16\left(\mathrm{dd}, J_{1}=13.9 \mathrm{~Hz}, J_{2}=8.1\right.$ $\mathrm{Hz}, 1 \mathrm{H}), 3.01\left(\mathrm{dd}, J_{1}=13.9 \mathrm{~Hz}, J_{2}=5.9 \mathrm{~Hz}, 1 \mathrm{H}\right), 2.86(\mathrm{q}, 1 \mathrm{H}), 2.01(\mathrm{~s}, 3 \mathrm{H}), 1.23(\mathrm{~d}, J=$ $6.9 \mathrm{~Hz}, 6 \mathrm{H}) ;{ }^{13} \mathrm{C} \mathrm{NMR}\left(\mathrm{CDCl}_{3}, 75 \mathrm{MHz}\right) \delta 170.2,147.1,140.3,134.4,129.4,128.3$, 127.9, 126.6, 126.3, 76.7, 42.5, 33.7, 24.0, 21.2; HRMS (EI) $\mathrm{C}_{19} \mathrm{H}_{22} \mathrm{O}_{2}$ calcd 282.1620 $\left(\mathrm{M}^{+}\right)$, found 282.1622 ; The \% ee was determined by HPLC on a $(R, R)$ Whelk-O1 $[n-$ hexane $/ 2$-propanol $=95 / 5$; flow rate $=1 \mathrm{~mL} / \mathrm{min}$; $U V=217 \mathrm{~nm}$; retention times $=7.6$ $\min (R), 5.4 \min (S)]$. 
(R)-1-Acetoxy-2-phenyl-1-(3-methylphenyl)-ethane $(3 \mathbf{3}):[a]_{\mathrm{D}}{ }^{25}=+28.6(c=1.0$, $\mathrm{CHCl}_{3}, 98 \%$ ee); ${ }^{1} \mathrm{H} \mathrm{NMR}\left(\mathrm{CDCl}_{3}, 300 \mathrm{MHz}\right) \delta 7.26-7.17(\mathrm{~m}, 4 \mathrm{H}), 7.12-7.07(\mathrm{~m}, 5 \mathrm{H})$, $5.91(\mathrm{t}, J=6.1 \mathrm{~Hz}, 1 \mathrm{H}), 3.17\left(\mathrm{dd}, J_{1}=13.8 \mathrm{~Hz}, J_{2}=8.2 \mathrm{~Hz}, 1 \mathrm{H}\right), 3.03\left(\mathrm{dd}, J_{1}=13.8 \mathrm{~Hz}\right.$, $\left.J_{2}=5.7 \mathrm{~Hz}, 1 \mathrm{H}\right), 2.33(\mathrm{~s}, 3 \mathrm{H}), 1.99(\mathrm{~s}, 3 \mathrm{H}) ;{ }^{13} \mathrm{C} \mathrm{NMR}\left(\mathrm{CDCl}_{3}, 75 \mathrm{MHz}\right) \delta 170.1,140.1$, 138.0, 137.2, 129.5, 128.7, 128.3, 128.2, 127.3, 126.5, 123.6, 76.7, 43.0, 21.4, 21.2; HRMS (EI) $\mathrm{C}_{17} \mathrm{H}_{18} \mathrm{O}_{2}$ calcd $254.1307\left(\mathrm{M}^{+}\right)$, found 254.1311; The \% ee was determined by HPLC on a $(R, R)$ Whelk-O1 $[n$-hexane/2-propanol $=95 / 5$; flow rate $=1 \mathrm{~mL} / \mathrm{min}$; $\mathrm{UV}=217 \mathrm{~nm}$; retention times $=10.5 \mathrm{~min}(R), 5.9 \mathrm{~min}(S)]$.

(R)-1-Acetoxy-2-phenyl-1-(3-methoxyphenyl)-ethane $(\mathbf{3 j}):[a]_{\mathrm{D}}{ }^{25}=+22.2(c=1.0$, $\mathrm{CHCl}_{3}, 98 \%$ ee); ${ }^{1} \mathrm{H} \mathrm{NMR}\left(\mathrm{CDCl}_{3}, 300 \mathrm{MHz}\right) \delta 7.26-7.16(\mathrm{~m}, 4 \mathrm{H}), 7.10(\mathrm{~d}, J=6.6 \mathrm{~Hz}$, 2H), $6.86(\mathrm{~d}, J=7.6 \mathrm{~Hz}, 1 \mathrm{H}), 6.80(\mathrm{~d}, J=6.2 \mathrm{~Hz}, 2 \mathrm{H}), 5.91(\mathrm{t}, J=6.4 \mathrm{~Hz}, 1 \mathrm{H}), 3.75(\mathrm{~s}$, $3 \mathrm{H}), 3.17\left(\mathrm{dd}, J_{1}=13.7 \mathrm{~Hz}, J_{2}=7.9 \mathrm{~Hz}, 1 \mathrm{H}\right), 3.03\left(\mathrm{dd}, J_{1}=13.7 \mathrm{~Hz}, J_{2}=6.0 \mathrm{~Hz}, 1 \mathrm{H}\right)$, $2.00(\mathrm{~s}, 3 \mathrm{H}) ;{ }^{13} \mathrm{C} \mathrm{NMR}\left(\mathrm{CDCl}_{3}, 75 \mathrm{MHz}\right) \delta 170.1,159.6,141.7,137.0,129.5,129.4$, 128.3, 126.6, 118.9, 113.4, 112.3, 76.5, 55.2, 43.0, 21.2; HRMS (EI) $\mathrm{C}_{17} \mathrm{H}_{18} \mathrm{O}_{3}$ calcd $270.1256\left(\mathrm{M}^{+}\right)$, found 270.1252 ; The \% ee was determined by HPLC on a $(R, R)$ WhelkO1 $[n$-hexane $/ 2$-propanol $=90 / 10$; flow rate $=1 \mathrm{~mL} / \mathrm{min}$; $\mathrm{UV}=217 \mathrm{~nm}$; retention times $=11.3 \min (R), 6.9 \min (S)]$.

(R)-1-Acetoxy-2-phenyl-1-(3-phenoxy)-ethane (3k): $[a]_{\mathrm{D}}{ }^{25}=-10.5\left(c=1.0, \mathrm{CHCl}_{3}\right.$, $99 \%$ ee); ${ }^{1} \mathrm{H}$ NMR $\left(\mathrm{CDCl}_{3}, 300 \mathrm{MHz}\right) \delta 7.34-7.19(\mathrm{~m}, 6 \mathrm{H}), 7.12-7.07$ (m, 3H), $7.00(\mathrm{~d}$, $J=7.6 \mathrm{~Hz}, 1 \mathrm{H}), 6.94-6.88(\mathrm{~m}, 4 \mathrm{H}), 5.89$ (t, $J=6.9 \mathrm{~Hz}, 1 \mathrm{H}), 3.17\left(\mathrm{dd}, J_{1}=13.7 \mathrm{~Hz}, J_{2}=\right.$ $7.7 \mathrm{~Hz}, 1 \mathrm{H}), 3.02\left(\mathrm{dd}, J_{1}=13.7 \mathrm{~Hz}, J_{2}=6.3 \mathrm{~Hz}, 1 \mathrm{H}\right), 2.01(\mathrm{~s}, 3 \mathrm{H}) ;{ }^{13} \mathrm{C} \mathrm{NMR}\left(\mathrm{CDCl}_{3}\right.$, $75 \mathrm{MHz}) \delta 170.0,157.1,157.0,142.1,136.8,129.7,129.5,129.4,128.3,126.6,123.3$, 121.4, 118.8, 118.3, 117.1, 76.2, 43.0, 21.1; HRMS (EI) $\mathrm{C}_{22} \mathrm{H}_{20} \mathrm{O}_{3}$ calcd $332.1412\left(\mathrm{M}^{+}\right)$, found 332.1411; The \% ee was determined by HPLC on a $(R, R)$ Whelk-O1 [ $n$-hexane/2propanol $=95 / 5$; flow rate $=1 \mathrm{~mL} / \mathrm{min} ; \mathrm{UV}=217 \mathrm{~nm}$; retention times $=13.4 \mathrm{~min}(R)$, $7.7 \mathrm{~min}(S)]$.

(R)-1-Acetoxy-2-phenyl-1-(4-fluorophenyl)-ethane (3I): $[a]_{\mathrm{D}}{ }^{25}=+30.3\left(c=1.0, \mathrm{CHCl}_{3}\right.$, $98 \%$ ee); ${ }^{1} \mathrm{H} \mathrm{NMR}\left(\mathrm{CDCl}_{3}, 300 \mathrm{MHz}\right) \delta 7.25-7.17(\mathrm{~m}, 5 \mathrm{H}), 7.06(\mathrm{~d}, J=6.3 \mathrm{~Hz}, 2 \mathrm{H})$, $6.98(\mathrm{t}, J=8.7 \mathrm{~Hz}, 2 \mathrm{H}), 5.90(\mathrm{t}, J=7.0 \mathrm{~Hz}, 1 \mathrm{H}), 3.19\left(\mathrm{dd}, J_{1}=13.7 \mathrm{~Hz}, J_{2}=7.6 \mathrm{~Hz}\right.$, $1 \mathrm{H}), 3.01\left(\mathrm{dd}, J_{1}=13.6 \mathrm{~Hz}, J_{2}=6.5 \mathrm{~Hz}, 1 \mathrm{H}\right), 2.02(\mathrm{~s}, 3 \mathrm{H}) ;{ }^{13} \mathrm{C} \mathrm{NMR}\left(\mathrm{CDCl}_{3}, 75 \mathrm{MHz}\right)$ $\delta 170.0,164.0,136.7,135.8,135.7,129.5,128.5,128.4,128.3,126.6,115.4,115.1$, 76.0, 42.9, 21.2; HRMS (EI) $\mathrm{C}_{16} \mathrm{H}_{15} \mathrm{O}_{2} \mathrm{~F}$ calcd 258.1056 $\left(\mathrm{M}^{+}\right)$, found 258.1059; The \% ee was determined by HPLC on a $(R, R)$ Whelk-O1 $[n$-hexane/2-propanol $=95 / 5$; flow rate $=1 \mathrm{~mL} / \mathrm{min} ; \mathrm{UV}=217 \mathrm{~nm}$; retention times $=9.2 \mathrm{~min}(R), 5.7 \mathrm{~min}(S)]$.

(R)-1-Acetoxy-2-phenyl-1-p-tolyl-ethane (3m): $[a]_{\mathrm{D}}{ }^{25}=+29.9\left(c=1.0, \mathrm{CHCl}_{3}, 98 \%\right.$ ee); ${ }^{1} \mathrm{H}$ NMR $\left(\mathrm{CDCl}_{3}, 300 \mathrm{MHz}\right) \delta 7.25-7.19(\mathrm{~m}, 5 \mathrm{H}), 7.16-7.09(\mathrm{~m}, 4 \mathrm{H}), 5.91(\mathrm{t}, J=$ 
$7.5 \mathrm{~Hz}, 1 \mathrm{H}), 3.19\left(\mathrm{dd}, J_{1}=13.7 \mathrm{~Hz}, J_{2}=7.9 \mathrm{~Hz}, 1 \mathrm{H}\right), 3.04\left(\mathrm{dd}, J_{1}=13.7 \mathrm{~Hz}, J_{2}=6.0\right.$ $\mathrm{Hz}, 1 \mathrm{H}), 2.32(\mathrm{~s}, 3 \mathrm{H}), 1.98(\mathrm{~s}, 3 \mathrm{H}) ;{ }^{13} \mathrm{C} \mathrm{NMR}\left(\mathrm{CDCl}_{3}, 75 \mathrm{MHz}\right) \delta 170.1,137.7,137.2$, 137.1, 129.5, 129.1, 128.2, 126.6, 126.5, 76.6, 42.9, 21.2, 21.1; HRMS (EI) $\mathrm{C}_{17} \mathrm{H}_{18} \mathrm{O}_{2}$ calcd 254.1307 $\left(\mathrm{M}^{+}\right)$, found 254.1310; The \% ee was determined by HPLC on a $(R, R)$ Whelk-O1 $[n$-hexane/2-propanol $=95 / 5$; flow rate $=1 \mathrm{~mL} / \mathrm{min} ; \mathrm{UV}=217 \mathrm{~nm}$; retention times $=10.9 \min (R), 6.2 \min (S)]$.

(R)-1-Acetoxy-2-phenyl-1-(4-methoxyphenyl)-ethane $(3 \mathrm{n}):[a]_{\mathrm{D}}^{25}=+32.5(c=1.0$, $\mathrm{CHCl}_{3}, 96 \%$ ee); ${ }^{1} \mathrm{H}$ NMR $\left(\mathrm{CDCl}_{3}, 300 \mathrm{MHz}\right) \delta 7.25-7.15(\mathrm{~m}, 5 \mathrm{H}), 7.09(\mathrm{~d}, J=6.7 \mathrm{~Hz}$, 2H), $6.83(\mathrm{~d}, J=8.6 \mathrm{~Hz}, 2 \mathrm{H}), 5.90(\mathrm{t}, J=7.0 \mathrm{~Hz}, 1 \mathrm{H}), 3.77(\mathrm{~s}, 3 \mathrm{H}), 3.19$ (dd, $J_{1}=13.7$ $\left.\mathrm{Hz}, J_{2}=7.8 \mathrm{~Hz}, 1 \mathrm{H}\right), 3.02\left(\mathrm{dd}, J_{1}=13.7 \mathrm{~Hz}, J_{2}=6.3 \mathrm{~Hz}, 1 \mathrm{H}\right), 1.98(\mathrm{~s}, 3 \mathrm{H}) ;{ }^{13} \mathrm{C} \mathrm{NMR}$ $\left(\mathrm{CDCl}_{3}, 75 \mathrm{MHz}\right) \delta 170.1,159.3,137.2,132.2,129.5,128.2,128.1,126.5,113.7,76.4$, 55.2, 42.8, 21.2; HRMS (EI) $\mathrm{C}_{17} \mathrm{H}_{18} \mathrm{O}_{3}$ calcd 270.1256 $\left(\mathrm{M}^{+}\right)$, found 270.1251; The \% ee was determined by HPLC on a $(R, R)$ Whelk-O1 $[n$-hexane/2-propanol $=90 / 10$; flow rate $=1 \mathrm{~mL} / \mathrm{min} ; \mathrm{UV}=217 \mathrm{~nm}$; retention times $=14.8 \mathrm{~min}(R), 7.0 \mathrm{~min}(S)]$.

(R)-1-Acetoxy-2-phenyl-1-(4-isopropylphenyl)-ethane $(\mathbf{3 o}):[a]_{\mathrm{D}}{ }^{25}=+27.8(c=1.0$, $\mathrm{CHCl}_{3}, 99 \%$ ee); ${ }^{1} \mathrm{H} \mathrm{NMR}\left(\mathrm{CDCl}_{3}, 300 \mathrm{MHz}\right) \delta 7.23-7.10(\mathrm{~m}, 9 \mathrm{H}), 5.93(\mathrm{t}, J=5.8 \mathrm{~Hz}$, $1 \mathrm{H}), 3.17\left(\mathrm{dd}, J_{1}=13.8 \mathrm{~Hz}, J_{2}=8.3 \mathrm{~Hz}, 1 \mathrm{H}\right), 3.03\left(\mathrm{dd}, J_{1}=13.8 \mathrm{~Hz}, J_{2}=5.6 \mathrm{~Hz}, 1 \mathrm{H}\right)$, $2.89(\mathrm{q}, 1 \mathrm{H}), 1.96(\mathrm{~s}, 3 \mathrm{H}), 1.23(\mathrm{~d}, J=6.9 \mathrm{~Hz}, 6 \mathrm{H}) ;{ }^{13} \mathrm{C} \mathrm{NMR}\left(\mathrm{CDCl}_{3}, 75 \mathrm{MHz}\right) \delta$ $170.1,148.7,137.5,137.4,129.5,128.3,126.7,126.5,126.4,76.5,43.0,33.9,24.0$, 21.2; HRMS (EI) $\mathrm{C}_{19} \mathrm{H}_{22} \mathrm{O}_{2}$ calcd $282.1620\left(\mathrm{M}^{+}\right)$, found 282.1616; The \% ee was determined by HPLC on a $(R, R)$ Whelk-O1 [ $n$-hexane/2-propanol $=95 / 5$; flow rate $=1$ $\mathrm{mL} / \mathrm{min} ; \mathrm{UV}=217 \mathrm{~nm}$; retention times $=7.8 \mathrm{~min}(R), 5.6 \mathrm{~min}(S)]$.

(R)-1-Acetoxy-2-phenyl-1-(2-naphthyl)-ethane (3p): $[a]_{\mathrm{D}}^{25}=+35.7\left(c=1.0, \mathrm{CHCl}_{3}\right.$, 98\% ee); ${ }^{1} \mathrm{H}$ NMR $\left(\mathrm{CDCl}_{3}, 300 \mathrm{MHz}\right) \delta 7.81-7.76(\mathrm{~m}, 3 \mathrm{H}), 7.71(\mathrm{bs}, 1 \mathrm{H}), 7.46-7.40(\mathrm{~m}$, $3 \mathrm{H}), 7.24-7.15(\mathrm{~m}, 3 \mathrm{H}), 7.12-7.10(\mathrm{~m}, 2 \mathrm{H}), 6.11(\mathrm{t}, J=7.2 \mathrm{~Hz}, 1 \mathrm{H}), 3.28\left(\mathrm{dd}, J_{1}=13.7\right.$ $\left.\mathrm{Hz}, J_{2}=7.8 \mathrm{~Hz}, 1 \mathrm{H}\right), 3.14\left(\mathrm{dd}, J_{1}=13.7 \mathrm{~Hz}, J_{2}=6.1 \mathrm{~Hz}, 1 \mathrm{H}\right), 2.02(\mathrm{~s}, 3 \mathrm{H}) ;{ }^{13} \mathrm{C} \mathrm{NMR}$ $\left(\mathrm{CDCl}_{3}, 75 \mathrm{MHz}\right) \delta 170.1,137.4,137.0,133.1,129.6,128.3,128.2,128.1,127.7,126.6$, 126.2, 126.1, 125.9, 124.4, 76.8, 42.9, 21.2; HRMS (EI) $\mathrm{C}_{20} \mathrm{H}_{18} \mathrm{O}_{2}$ calcd 290.1307 (M+), found 290.1308; The \% ee was determined by HPLC on a $(R, R)$ Whelk-O1 [ $n$-hexane/2propanol $=90 / 10$; flow rate $=1.5 \mathrm{~mL} / \mathrm{min} ; \mathrm{UV}=217 \mathrm{~nm}$; retention times $=26.4 \mathrm{~min}$ $(R), 5.5 \min (S)]$.

\section{Preparation of $\left(\eta^{5}-\mathrm{Ph}_{4} \mathrm{C}_{4} \mathrm{COBz}\right) \mathrm{Ru}(\mathrm{CO})_{2} \mathrm{Cl}(5)$.}

Method A: A solution of $\eta^{4}-\left(\mathrm{Ph}_{4} \mathrm{C}_{4} \mathrm{CO}\right)(\mathrm{CO})_{3} \mathrm{Ru}(6)(200 \mathrm{mg}, 0.351 \mathrm{mmol})$ and benzoyl chloride $(50 \mathrm{mg}, 0.35 \mathrm{mmol})$ in toluene $(4 \mathrm{~mL})$ was placed in a $50-\mathrm{mL}$ flask equipped with a grease-free high-vacuum stopcock under argon. The mixture was 
heated at $80^{\circ} \mathrm{C}$ for $6 \mathrm{~h}$ under argon. The reaction mixture was concentrated and purified by chromatography on a silica-gel column to give pale yellow solid 5 (228 mg, 95\% yield). m.p. $142-145{ }^{\circ} \mathrm{C}$ (dec.); ${ }^{1} \mathrm{H}$ NMR $\left(\mathrm{CDCl}_{3}, 300 \mathrm{MHz}\right) \delta 7.95$ (d, $J=7.3 \mathrm{~Hz}, 2 \mathrm{H}$, Ar), $7.58(\mathrm{~m}, 1 \mathrm{H}, \mathrm{Ar}), 7.04-7.43(\mathrm{~m}, 22 \mathrm{H}, \mathrm{Ar}) ;{ }^{13} \mathrm{C} \mathrm{NMR}\left(\mathrm{CDCl}_{3} ; 75 \mathrm{MHz}\right) \delta 196.5$ (CO), $164.3(\underline{\mathrm{C}}(=\mathrm{O}) \mathrm{Ph}), 134.8,132.5,131.3,130.7,129.2,129.1,129.0,128.8,128.7$, 128.3, 128.1, 127.8, 127.6, $104.8\left(\mathrm{C}_{2}, \mathrm{C}_{5}\right.$ in $\mathrm{Cp}$ ring), $95.2\left(\mathrm{C}_{3}, \mathrm{C}_{4}\right.$ in $\mathrm{Cp}$ ring); $\mathrm{IR}(\mathrm{KBr}$, $\left.\mathrm{cm}^{-1}\right): \quad v(\mathrm{CO})=2045$ (s), 1998 (s) 1747 (s); element analysis (\%) calcd for $\mathrm{C}_{38} \mathrm{H}_{25} \mathrm{ClO}_{4} \mathrm{Ru}$ : C, 66.91; $\mathrm{H}, 3.69$; found: $\mathrm{C}, 66.79$; $\mathrm{H}, 3.67$.

Method B: A solution of $\left[\left(\eta^{5}-\mathrm{Ph}_{4} \mathrm{C}_{4} \mathrm{CO}\right) \mathrm{Ru}(\mathrm{CO})_{2}\right]_{2}(7)(200 \mathrm{mg}, 0.185 \mathrm{mmol})$ and benzoyl chloride $(54.8 \mathrm{mg}, 0.390 \mathrm{mmol})$ in toluene $(4 \mathrm{~mL})$ was placed in a $50-\mathrm{mL}$ flask equipped with a grease-free high-vacuum stopcock under argon. The mixture was stirred at room temperature for $2 \mathrm{~h}$ under argon. The reaction mixture was concentrated and purified by chromatography on a silica-gel column to give 5 ( $245 \mathrm{mg}, 92 \%$ yield).

\section{Hydrolysis of $(\boldsymbol{R})$-acetoxy-1,2-diphenylethane (3a)}

A mixture of $3 \mathbf{a}\left(100 \mathrm{mg}, 0.416 \mathrm{mmol}, 98 \%\right.$ ee) and $\mathrm{K}_{2} \mathrm{CO}_{3}$ (2.0 equiv. $0.832 \mathrm{mmol}$, $115 \mathrm{mg})$ in $1 \mathrm{~mL} \mathrm{MeOH} / \mathrm{H}_{2} \mathrm{O}=4 / 1(\mathrm{v} / \mathrm{v})$ solvent was stirred in a $10 \mathrm{~mL}$ reaction vessel at the room temperature for 1 hour. The completion of the reaction was checked by TLC and then evaporated solvents. The reaction mixture was poured into $\mathrm{H}_{2} \mathrm{O}$ and extracted with $\mathrm{CH}_{2} \mathrm{Cl}_{2}(10 \mathrm{~mL} \times 3)$. The combined organic layer was washed with brine, dried over anhydrous $\mathrm{MgSO}_{4}$. The crude compound was purified on a $\mathrm{SiO}_{2}$ short column chromatography to give $(R)-\mathbf{2 a}$ (white solid, $81 \mathrm{mg}, 98 \%$ yield, $98 \%$ ee): m.p. $69-70{ }^{\circ} \mathrm{C}$ (lit. 66-70 $\left.{ }^{\circ} \mathrm{C}\right) ;[\alpha]_{\mathrm{D}}{ }^{26.9}=-53.8\left(c=1.0\right.$, EtOH, 98\% ee) $\left(\right.$ lit. $[\alpha]_{\mathrm{D}}{ }^{23}=-52.9(c=1.0$, $\mathrm{EtOH},>98 \%$ ee); The ${ }^{1} \mathrm{H}$ and ${ }^{13} \mathrm{C}$ NMR data were in good agreement with those reported in the literature (lit.: David, C.; Paul, H.; Julian, P. H.; Ian, C. L.; Graham, M.; Paul, M.; Christopher, J. P.; James, A. R.; Simon, W.; Antonio, Z-G. Org. Proc. Res. Dev., 2003, 7, 89-94.); The \% ee was determined by HPLC on a Chiralcel OD-H column (Daicel) $[n$-hexane $/ 2$-propanol $=90 / 10$; flow rate $=1 \mathrm{~mL} / \mathrm{min} ; \mathrm{UV}=217 \mathrm{~nm}$; retention times $=8.3 \mathrm{~min}(R), 9.5 \min (S)]$. 